\title{
Kaposi's Sarcoma of the Foot: A Case Report
}

\author{
by Al Kline, DPM 1 四
}

The Foot \& Ankle Journal 1 (3): 1

A case of ulcerated, classic Kaposi Sarcoma (KS) of the foot is described. This is a tumor of vascular endothelial origin which most commonly present in men 50-70 years of age. This case report includes presentation and discussion of differential diagnosis, surgical removal, histopathology interpretation, incidence of metastasis and treatment.

Key words: Kaposi’s sarcoma, metastasis, ulcerated KS, classic KS, pedunculated KS

Accepted: February 10, 2008

Published: March 1, 2008

This is an Open Access article distributed under the terms of the Creative Commons Attribution License. It permits unrestricted use, distribution, and reproduction in any medium, provided the original work is properly cited. @The Foot \& Ankle Journal (www.faoj.org)

Kaposi's sarcoma (KS) was first described by Hungarian dermatologist Moritz Kaposi in 1872. ${ }^{1,2}$ Kaposi first described the lesions as "idiopathic, multiple pigmented sarcoma of the skin". Kaposi's sarcoma is a spindle cell tumor of endothelial origin. It is commonly associated with the endothelial lining of blood vessels. In the early 1980's, KS was commonly associated with the AIDS epidemic. Four types of Kaposi's sarcoma have been described that include epidemic AIDS related Kaposi's Sarcoma, Immunocompromised KS, Endemic African KS and Classic KS. The classic KS is the only form not associated with the AIDS epidemic. In the past two decades, with the improvement in AIDS medication and treatment, the incidence of AIDS related KS has decreased by $90 \%{ }^{1}$

\footnotetext{
Address correspondence to: Dr. Al Kline, DPM, 3130 South Alameda, Corpus Christi, Texas 78404. Email: Al@Kline.net

${ }^{1}$ Adjunct Clinical Faculty, Barry University School of Podiatric Medicine. Private practice, Chief of Podiatry, Doctors Regional Medical Center. Corpus Christi, Texas, 78411.
}

Classic KS was first described as most prevalent in elderly Jewish, Mediterranean men between the ages of 50-70 years. ${ }^{1,3}$ The ratio of KS in men to women is $15: 1{ }^{1}$ The highest incidence of $\mathrm{KS}$ is reported in Sicily.

In the lower extremity, the lesion is most commonly associated with venous stasis and lymphedema. However, lesions on the plantar surface of the foot are rarely associated with edema which complicates this classic description. The lesions are usually violasceous, red to pink in color. Almost all cutaneous lesions are raised, non-pruritic and symmetrical. The histopathology of KS reveals excessive spindle-cell proliferation throughout the endothelium. Human Herpes Virus 8 (HHV-8) has been identified in over 90\% of all KS tumors, suggesting a causative role. ${ }^{2}$ The incidence of KS of the foot is rarely reported. This report describes a case of ulcerated, classic Kaposi's sarcoma of the foot is described. 


\section{Case Report}

A 71 year old male presents to our office on consultation with a "growth" to the bottom of the left foot. (Figs. 1abc) The patient and family report he has had this lesion for a number of years. It recently became more macerated with 'bleeding' due to its size and location on the bottom of the foot. The patient has no history of allergies. His medications include Gabapentin, Metformin, Trental, Glipizide, Metaprolol and Lisinopril. He has type 2 diabetes, hypertension and has undergone previous vein stripping. His physical examination reveals a well nourished male with palpable pulses. Minimal edema was noted to his extremities with some pretibial pitting edema.

The patient has been covering the growth with a large, occlusive bandage that caused significant maceration of the lesion. The patient also had a smaller lesion along the medial distal foot. The patient had the lesion on the bottom of the foot for over two years without discomfort. The lesion is macerated and vascular in appearance, pedunculated and attached to a 'stalk'. (Fig. 2) MRI of the foot revealed a cutaneous soft tissue growth not extending into subcutaneous fat. (Fig. 3) The patient was taken to surgery to remove both lesions by simple excision.

The patient was brought to the operating room and under local, IV sedation, the lesions were elipsed and removed. Simple horizontal mattress sutures were used to close the excision sites. A simple dressing was dispensed and the region healed without complication. To date, there has been no recurrence of the tumors.

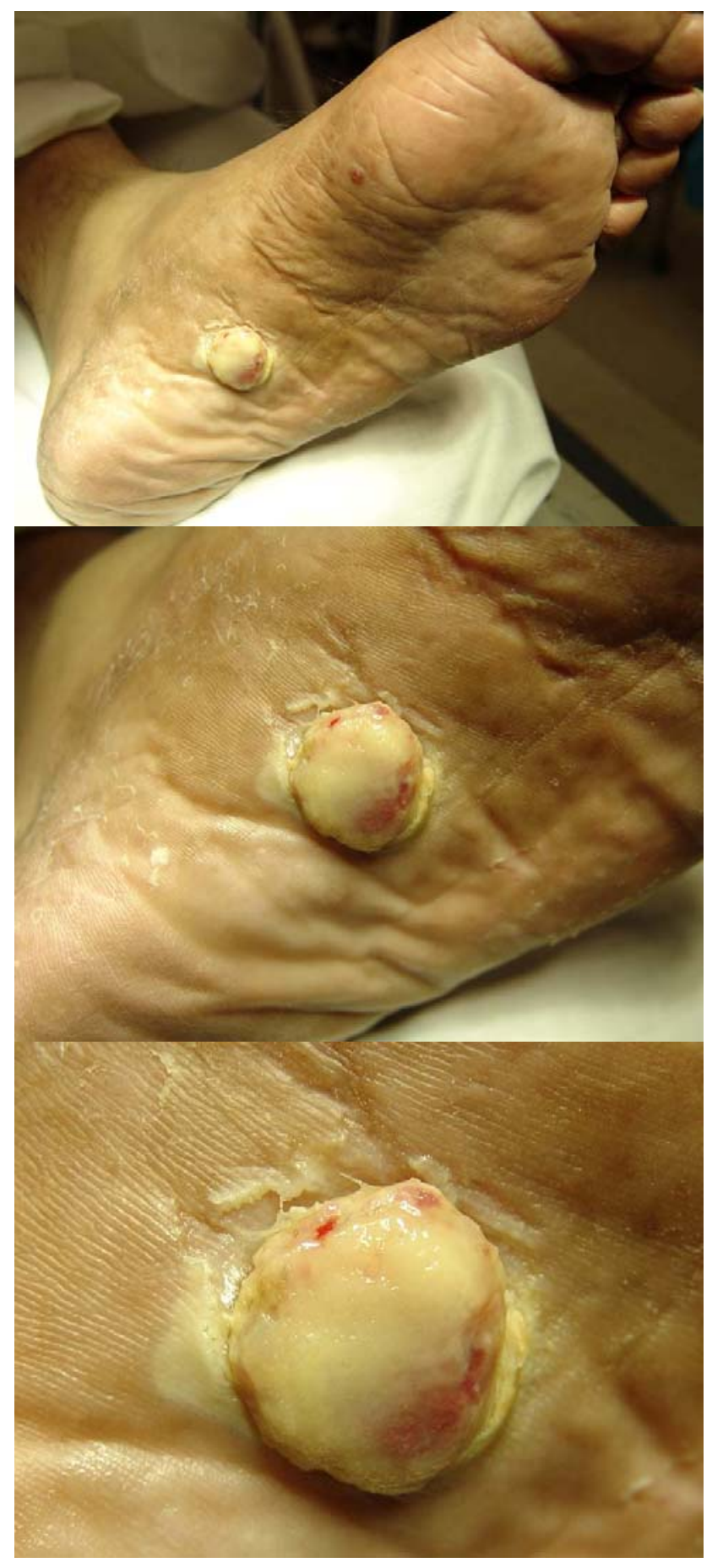

Figures 1abc The Kaposi's tumor measuring $30 \mathrm{~mm}$ in diameter. This circular soft tissue tumor of endothelial origin is macerated from occlusive dressings placed over the lesion. A smaller lesion is noted along the plantar, medial border of the foot along the first MPJ . 


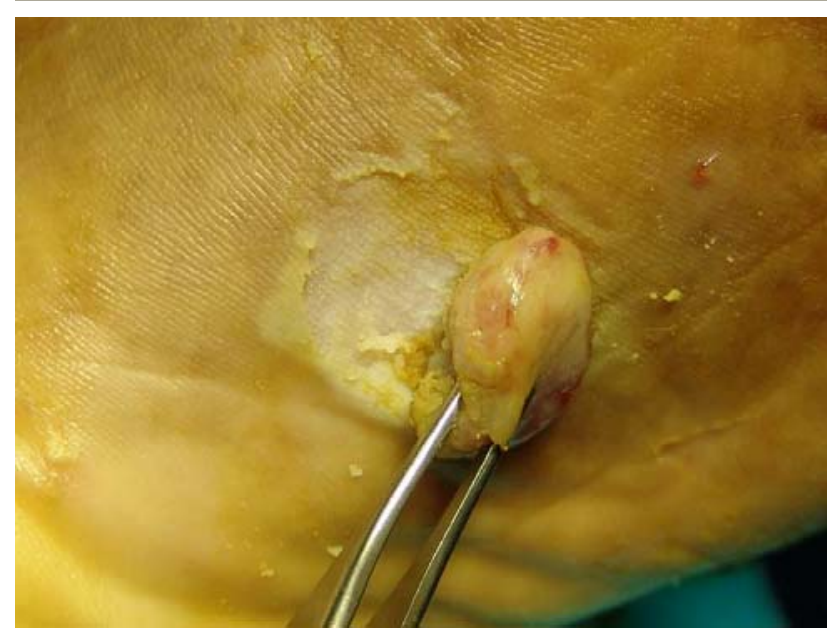

Figure 2 The tumor is attached to a stalk or pedicle.

\section{Histopathology}

The histological characteristic of KS does not vary between the different clinical types, but the progressive nature of the tumor varies. Early lesions show spindle cell proliferation within the interstitium of the upper dermis close to the vascular plexus. ${ }^{4}$ Nodular lesions are characteristic of older and more progressive tumors.

The surgical specimens were sent in formalinfilled containers. Microscopic sections and stepsections show a biopsy of acral skin and ulceration. In the dermis, there is a nodular dermal proliferation of atypical spindle cells arranged in nests and fascicles. (Fig. 4) Within the spindle cell proliferation, there are slit-like vascular spaces with extravasation of red blood cells. (Fig. 5) Slit-like vascular spaces containing erythrocytes are typical of the histologic characteristics of KS. These split-like spaces with extravasation sporadically display features of lymphangioma. ${ }^{4}$ Immunoperoxidase stains consisting of HHV-8 and S-100 were performed at the Baylor College of Medicine reference laboratory. This report confirms atypical spindle cells demonstrating nuclear positivity for HHV-8 and negative for S-100. This renders the final diagnosis as ulcerative Kaposi's sarcoma.

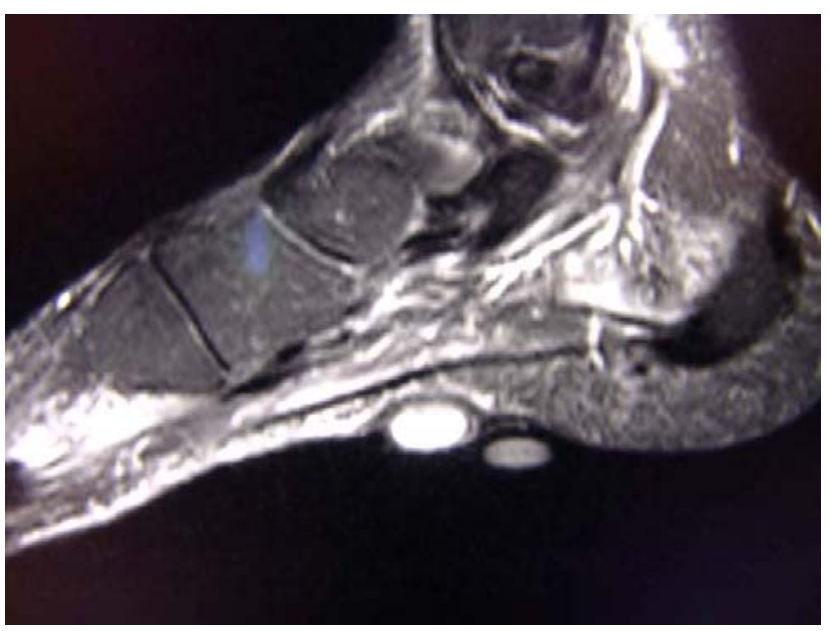

Figure $\mathbf{3}$ The pedunculated tumor is located in the acral skin with nodular proliferation of the dermis.

\section{Discussion}

Our case describes an isolated, pedunculated and ulcerated classic Kaposi's sarcoma of the foot with a smaller secondary lesion. The ulcerated type of KS appears to be rare with infrequent reports in the literature. The etiology of this type of lesion is unknown. The tumor has been associated with venous stasis and lymphedema. Our patient had signs of venous congestion and lymphedema, but the tumors location is atypical of this association. In recent studies, the tumor is now associated with human herpesvirus- 8 (HVV8 ) or commonly called Kasposi sarcoma herpesvirus (KSHV). ${ }^{2,4}$

It is one of the few viruses now proven to be associated with tumorigenesis in humans. ${ }^{4}$ The lesions also appear to be associated with immunosuppression. It could be theorized that classic KS arises in elderly men from some form of immunosuppression or compromise. For example, patients who undergo organ transplants and take rejection drugs to suppress the immune system are 150-200 times more likely to 'acquire' Kaposi's sarcoma. ${ }^{1}$ 


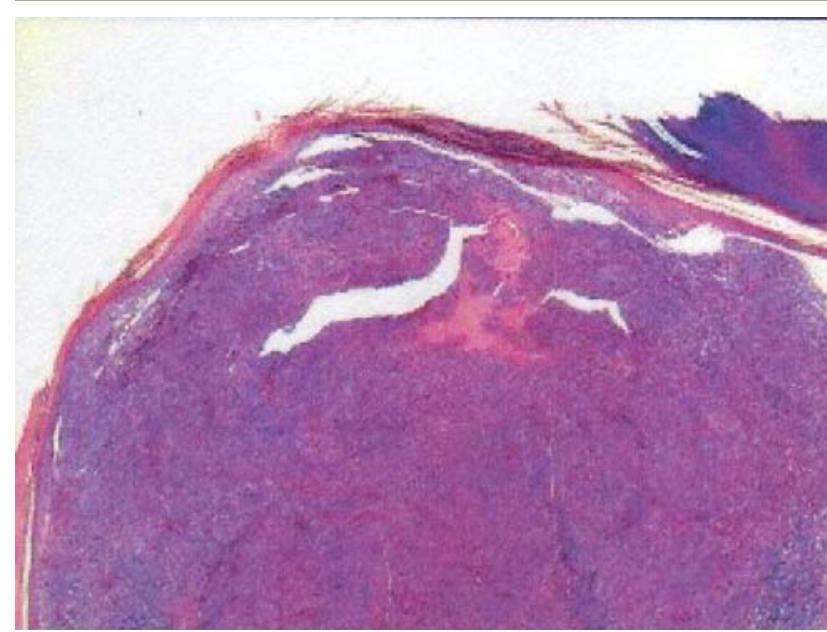

Figure 4 Under simple H\&E (Hematoxylin-eosin) stain, magnification $\times 20$. Lesional cells stained positive with CD31 and focally with CD34.

Commonly, the cutaneous lesions of KS are more diffuse and violasceous in color and distribution. However, the clinical presentation can vary greatly. The differential diagnosis should include acroangiodermatitis, port wine stains of the extremities, Sturge Weber Syndrome, nodular malignant melanoma, angiosarcoma, hemangioma, dermatofibroma, pyogenic granuloma or purpura. ${ }^{1,2,3}$

Treatment of classic KS can vary from simple surgical removal of the lesions to radiation treatment. If lesions are solitary, as in this case, surgical excision of the lesion is the best form of treatment. Brenner, et al, reported 52 patients who underwent primary excision for solitary lesions, $56 \%$ had no recurrence of lesions in a median of 15 months. ${ }^{8}$ If the classic KS is diffuse, irradiation of water while the legs are submerged in a water tank appears to be an accepted treatment. ${ }^{3}$ However, recurrence of lesions seems to be more common when the lesions are diffuse. Isolated lesions are best treated by excision, laser and surgical cauterization. Intralesional interferon injection is still considered controversial. ${ }^{4}$ Cryotherapy can be used for superficial lesions but are likely to recur. ${ }^{3}$

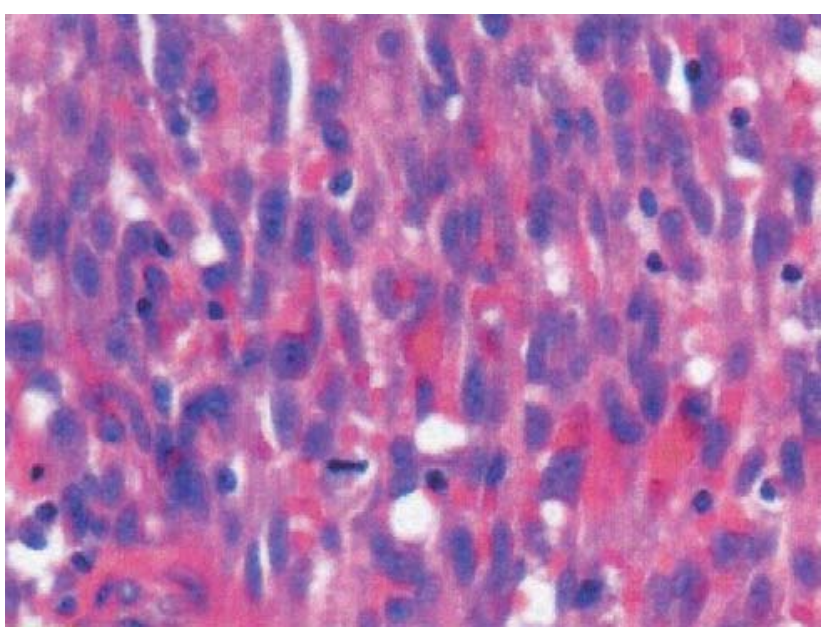

Figure 5 High power view of lesional cells. Note extravasation of red blood cells in slit-like spaces, magnification $\times 400$.

In one report, cyrotherapy has been shown to be $70 \%$ effective for superficial lesions. ${ }^{4}$ Occasionally, the lesions and disease may regress spontaneously. ${ }^{7}$

Incidence of metastasis varies in the literature. Some report that classic KS rarely metastasize. ${ }^{1}$ Another report suggests that up to one-third of patients with classic KS develop a secondary primary malignancy, most often non-Hodgkins lymphoma. ${ }^{3}$ Other reports suggest that over 35\% of patients with the disease of more than 15 years may develop secondary malignancies such as leukemia, myeloma and lymphomas. ${ }^{4,5,6}$ It appears that cases should be handled individually and risk should be assessed by the number of lesions and their location. Classic KS rarely disseminate and cause visceral or mucous membrane tumors. Dissemination is seen in less than $10 \%$ of the patients with KS. ${ }^{4}$ Certainly, if widespread visceral involvement is diagnosed, combination treatment including Chemotherapy, radiation treatment, surgical excision of lesions and conservative care is recommended. ${ }^{4}$ 


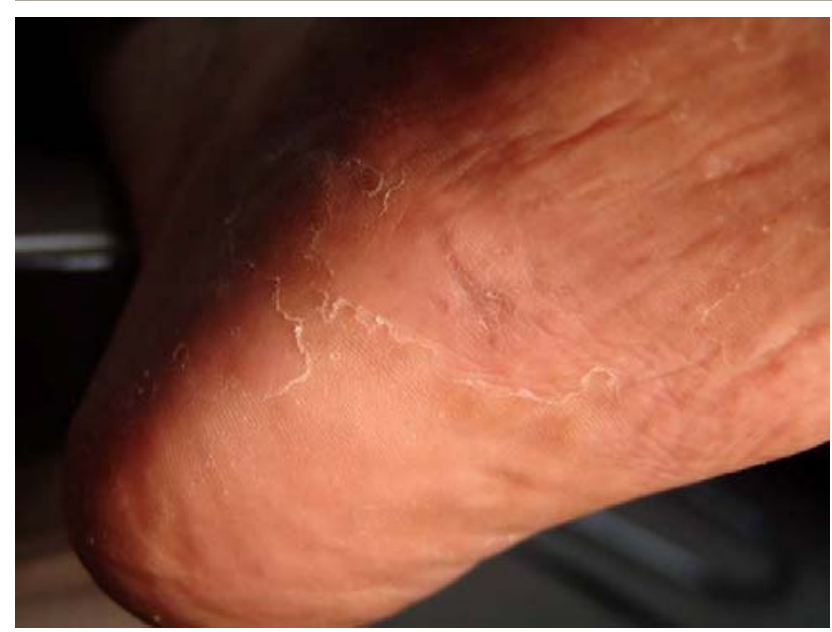

Figure 6 A few weeks after excision of tumor.

This report describes an unusual case of ulcerated Kaposi's sarcoma of the foot. Simple excision of the lesion was recommended without further cutaneous or systemic treatment. A smaller lesion on the foot was also removed which was consistent with KS. The surgeon has a variety of options for treatment of such lesions. In this case, surgical excision was the procedure of choice and the region healed without complication. (Fig. 6) Inspection of the patients mucous membranes revealed no other suspected, discolored or violasceous plaques. We have recommended the patient undergo routine colonoscopy to rule out any visceral involvement.

\section{References:}

1. American Cancer Society: What is Kaposi Sarcoma, [online], 2008.

2. Fishman, A., Sparano, D.: Kaposi Sarcoma. eMedicine [online], 2008.

3. Dubilier, L.D., Joffe, E.: Case report: Classic Kaposi's sarcoma, JAAPA, 18 (7), July, 2005. [DF]

4. Phillips, T.J.: Kaposi's Sarcoma,Wounds 13 (6): 237-240, 2001. [Online]

5. Piette WW. The incidence of second malignancies in subsets of Kaposi's sarcoma. J Am Acad Dermatol :16:855, 1987.

6. Safai B, Goo RA. Kaposi's sarcoma: A review and recent developments. Cancer: 44:419-33. 1981.

7. Chang Y, Cesarman E, Pessin MS, et al. Identification of herpesvirus-like DNA sequences in AIDS-associated Kaposi's sarcoma. Science 266: 1865-1869, 1994.

8. Brenner B, Rakowsky E, Katz A, Gutman H, et al. Tailoring treatment for classical Kaposi's sarcoma: Comprehensive clinical guidelines. Int $\mathrm{j}$ Oncol: 14 (6), 1097-1102, 1999.

Special thanks to Dr. Tu H Nguyen, MD, Board certified in Anatomic Pathology, Dermatopathology, and Cytopathology in preparing the slides. (Quest Diagnostics) 